Original Research

\title{
Experimental Effects of Acute Exercise in Attenuating Concurrent Memory Interference
}

\author{
Hannah Lewandowski, Lindsay Crawford, Paul D. Loprinzi *
}

Exercise \& Memory Laboratory, Department of Health, Exercise Science and Recreation Management, The University of Mississippi, University, MS 38677, USA; E-Mails: hjlewan1@go.olemiss.edu; Icrawfor@go.olemiss.edu; pdloprin@olemiss.edu

* Correspondence: Paul D. Loprinzi; E-Mail: pdloprin@olemiss.edu

Academic Editor: Gerhard Litscher

OBM Integrative and Complementary Medicine 2019, volume 4, issue 2

doi:10.21926/obm.icm.1902039
Received: April 13, 2019

Accepted: June 24, 2019

Published: June 25, 2019

\begin{abstract}
Acute exercise may enhance episodic memory recall, or the retrospective recall of an event or episode due to exercise-induced neuronal excitability and ensuing long-term potentiation. Of interest to this paper is the potential effects of acute exercise on memory interference, when the interfering stimuli occurs at the same time as the target stimuli. A three-arm, parallel-group randomized controlled intervention was employed. Participants $(\mathrm{N}=50)$ were randomized into one of three groups, including 1) Interference + Exercise, 2) Interference Only, and 3) Control (no interference and no exercise). The Interference component refers to the concurrent memory interference stimuli. The experimental conditions, although different for each group, included exercising at $70 \%$ of heart rate max, completing an online Sudoku puzzle during rest periods, completing a computerized Stroop task (word-color), listening to a pre-recorded 15word list, and subsequently recalling the words after observing a 20-minute video clip. For the memory assessment, the Control condition (i.e., no interference and no exercise) had significantly higher immediate memory ( 7.76 words) when compared to the interference +
\end{abstract}

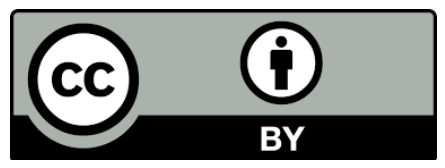

(C) 2019 by the author. This is an open access article distributed under the conditions of the Creative Commons by Attribution License, which permits unrestricted use, distribution, and reproduction in any medium or format, provided the original work is correctly cited. 
Exercise $(\mathrm{P}=0.0003 ; 4.70$ words) and Interference $(\mathrm{P}<0.0001 ; 3.93$ words) groups. For delayed memory recall, the Control group recalled more words than the Interference + Exercise $(P=0.001)$ and Interference groups $(P=0.0003)$, but there was no statistically significant difference between Interference + Exercise and Interference only $(P=0.24)$. These results provide strong evidence for a concurrent memory interference effect. We did not, however, demonstrate statistical evidence that acute moderate-intensity exercise attenuates a concurrent memory interference effect.

\section{Keywords}

Memory; proactive interference; retroactive interference; concurrent memory interference; prefrontal cortex; Stroop Task

\section{Introduction}

Recent work from our laboratory demonstrates that acute exercise may enhance episodic memory recall [1-5], or the retrospective recall of an event or episode [6]. We have detailed the mechanisms of this effect elsewhere [7-9], which includes, for example, exercise-induced neuronal excitability and ensuing long-term potentiation [10]. Of interest to this paper, written as a brief report, is the potential effects of acute exercise on memory interference. Typically, memory interference is subdivided into either proactive or retroactive memory interference. Proactive memory interference occurs when a previously learned memory interferes with the learning process of a subsequent memory stimulus. Conversely, retroactive interference occurs when newly acquired information interferes with previously acquired information.

It is conceivable that acute moderate-intensity exercise may help to minimize a memory interference effect. Memory interference appears to be influenced by the medial prefrontal cortex, as this structure may attenuate a memory interference effect via encoding (facilitating pattern separation) and retrieval (facilitating inactivation of the interfering engram) mechanisms [11]. To our knowledge, only three published experiments have evaluated the effects of acute exercise on attenuating cognitive-related memory interference [12-14], as opposed to motor-related memory interference $[15,16]$. These experiments come from our laboratory and demonstrate some, albeit limited, evidence that exercise may help to attenuate a memory interference effect. We speculate that this potential effect comes from exercise-induced alterations in prefrontal cortex function [11, 17-19].

Adding additional novelty to this line of inquiry, the present experiment evaluates whether acute moderate-intensity exercise can attenuate a concurrent interference effect, which has yet to be investigated. That is, herein we examined whether acute exercise can attenuate an interference effect when the interfering stimuli occurs at the same time as the target stimuli. This contrasts with proactive (interfering stimuli occurs before target stimuli) and retroactive interference (interfering

stimuli comes after the target stimuli). We hypothesize that acute exercise will attenuate a 
concurrent memory interference effect. Such an effect is plausible, as, in theory, working memory capacity may play a critical role in attenuating concurrent memory interference, and acute exercise has been shown to enhance working memory capacity [20].

\section{Methods}

\subsection{Study Design}

A three-arm, parallel-group randomized controlled intervention was employed. This study was approved by the authors' institutional review board. All participants provided written consent prior to participation.

Participants were randomized into one of three groups, including 1) Interference + Exercise, 2) Interference Only, and 3) Control (no interference and no exercise). The interference component refers to the concurrent memory interference stimuli. Further details are described below.

\subsection{Participants}

In total, 50 participants were recruited, including 17, 16, and 17 in the Interference + Exercise, Interference Only, and Control groups, respectively. Recruitment occurred via a convenience-based, non-probability sampling approach (classroom announcement and word-of-mouth). Participants included undergraduate and graduate students between the ages of 18 and 35 yrs.

Additionally, participants were excluded if they:

Self-reported as a daily smoker $[21,22]$

Self-reported being pregnant [23]

Exercised within 5 hours of testing [24]

Consumed caffeine within 3 hours of testing [25]

Had a concussion or head trauma within the past 30 days [26]

Took marijuana or other illegal drugs within the past 30 days [27]

Were considered a daily alcohol user (>30 drinks/month for women; >60 drinks/month for men) [28]

\subsection{Experiment Protocols}

A schematic of the experimental protocol is shown in Figure 1 and detailed hereafter.

Interference + Exercise. Participants exercised for 15-minutes on a treadmill (details described below). Afterward, they rested (seated) for 5-minutes while playing Sudoku (details described below). After this 5-minute rest period, we employed a cognitive paradigm that resembles the dual-task paradigm. Participants completed a computerized Stroop task (word-color). This task took approximately 3-minutes to complete. They first completed this entire task as a familiarization trial. After completing the familiarization trial, they re-completed the task. At the 30 -second point into this task, they listened to a pre-recorded list of 15 -words (1.5 seconds per word) via headphones. After a 10-second delay, the re-listened to the list a second time and then immediately performed a freerecall (short-term memory). Following this, they finished completing the Stroop task. Afterward, they 
watch a video clip ("The Office - Bloopers") for 20-minutes (as a distractor) and then were asked to recall as many words as possible from the word list.

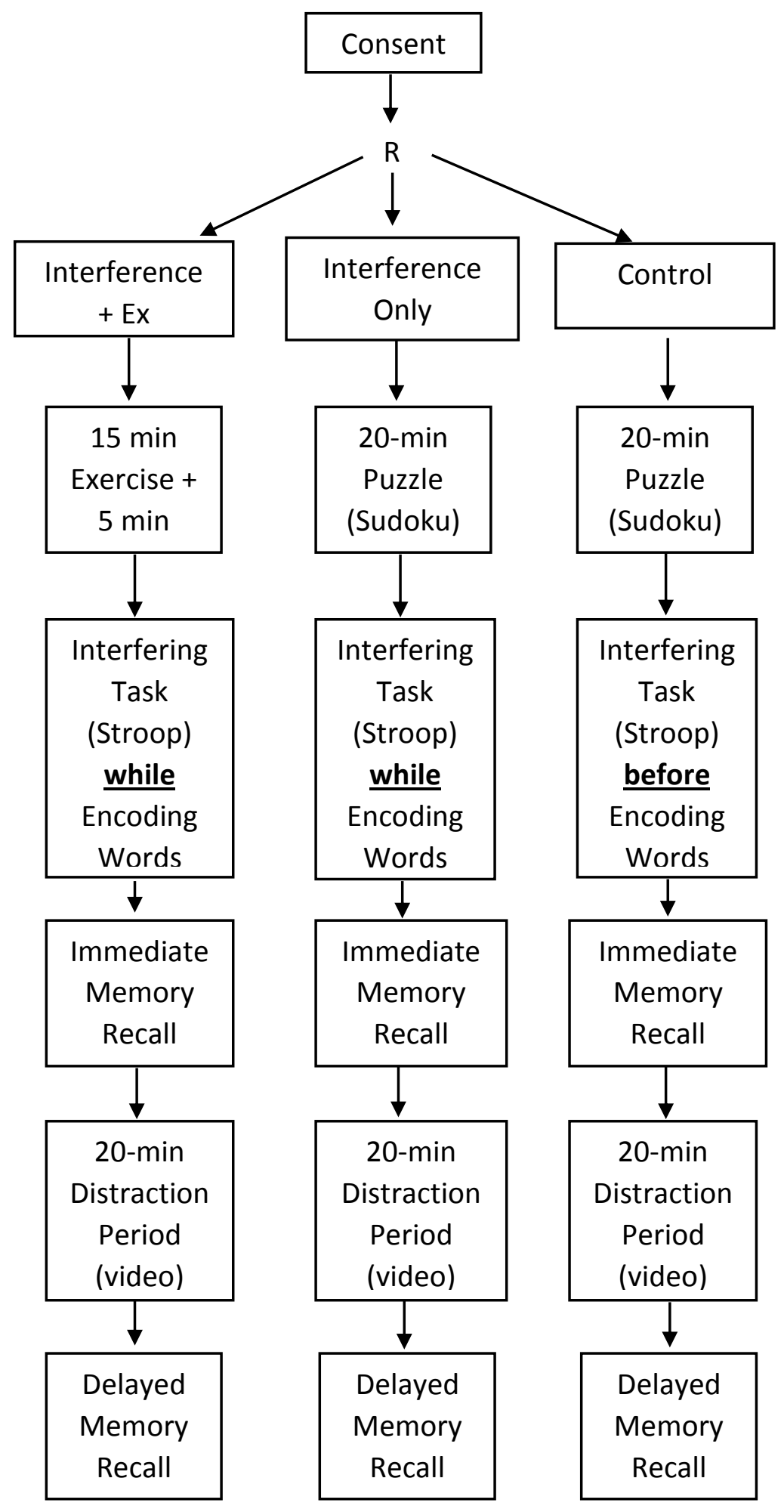

Figure 1 Schematic of the study protocol.

$$
R=\text { Randomization; } \mathrm{Ex}=\text { Exercise }
$$


Notably, at the beginning of the task, they were instructed as follows: "You are now going to complete a practice trial of the Stroop task. It should take approximately 3minutes. After you complete this practice trial, you will re-complete this task. While completing this task the second time, you will hear a recording of 15-words through the headphones. This recording will play twice. Please do your best to try and remember as many of these words as possible, as later in the experiment you will be asked to recall these words. It is also important that you do your best on the Stroop task. Thus, try and focus on both tasks (Stroop and listening to the words) at the same time. Both tasks are of equal importance as we will be evaluating your scores on both tasks."

Interference Only. This group completed the same protocol as the Interference + Exercise Group, with the exception that they did not exercise. Instead of exercising for 15 -minutes followed by a 5minute rest period (above group), they played Sudoku for 20-minutes before starting the Stroop task.

No Interference and No Exercise (control). This group played Sudoku for 20-minutes. Then they completed two rounds of the Stroop task (results from the second Stroop task were recorded). After completing the second Stroop task, they listened to the word list twice (10-sec break). After listening to the word lists, they performed an immediate free-recall and then watched a video clip ("The Office - Bloopers") for 20-minutes. Following this, they were asked to recall as many words as possible from the word list.

\subsection{Exercise Protocol}

Participants randomized to the exercise group exercised (brisk walk) on a treadmill for 15 minutes. Participants exercised at approximately $70 \%$ of their estimated HR max (220-age), which constitutes moderate-intensity exercise [29]. The target heart rate was calculated prior to the exercise bout and the treadmill incline and speed were altered at the very beginning of the exercise to ensure heart rate approached the target level within the first few minutes of exercise.

Immediately after the bout of exercise, participants rested in a seated position for 5 minutes. Similar to other studies [30], during this resting period, they played on-line game of Sudoku (described below) to prevent boredom. After this resting period, they commenced the memory assessment, as described below.

\subsection{Control Protocol}

The Sudoku task involved a medium-level, on-line administered, Sudoku puzzle. The website for this puzzle is located here: https://www.websudoku.com/

\subsection{Memory Assessment}

Word List Task. 15 words were extracted from the Toronto Word Pool, all with imagery scores $>5$. Participants listened to these words, delivered at a rate of 1 word per 1.5 seconds, via headphones. Previous research demonstrates evidence of test-retest reliability for related word-list memory tasks [31]. 
Stroop Task. The Stroop color word test is a well-documented prefrontal activation task indicative of executive function [32], which demonstrates adequate psychometric properties [33]. Testing of the Stroop effect was performed using computerized software. Specifically, the color word Stroop testing with keyboard responding was used. Participants were given color words (e.g. "red") written in color and asked to indicate the color of the word (not its meaning) by specific key presses. They were instructed to accomplish this as quickly and accurately as possible. There were 84 total trials, consisting of 4 colors (red, green, blue, black) x 3 color-stem congruency (congruent, incongruent, control) $x 7$ repetitions. The stimuli remained on the screen until the key response, with latencies measured from the onset of the stimuli. The congruent trials involved the color word and the color it presented being the same; incongruent trials involved the color word being different than the color it was presented in (e.g., it read GREEN, but this word was not in the green color); and the control trials involved colored rectangles. The outcome measure was the average latency (in milliseconds [ms]) of the correctly identified congruent, incongruent and control trials.

\subsection{Additional Assessments}

Various demographic (e.g., BMI), behavioral (e.g., habitual physical activity and resistance exercise) and psychological (e.g., RPE) assessments were completed to ensure that the groups were similar on these parameters. As a measure of habitual physical activity behavior, participants completed the Physical Activity Vital Signs Questionnaire to evaluate time spent per week in moderate-to-vigorous physical activity (MVPA) [34]. Height/weight (BMI; $\mathrm{kg} / \mathrm{m}^{2}$ ) were measured to provide anthropometric characteristics of the sample. Lastly, before and after the exercise and control conditions, heart rate (chest-strapped Polar monitor, F1 model) was assessed.

\subsection{Statistical Analysis}

All statistical analyses were computed in Stata (v. 12). A one-way ANOVA was used to compare the Stroop scores across the three groups, with Bonferroni-corrected post-hoc tests employed. A oneway repeated measures ANOVA was employed for the word-list task. Main effects for time and group, as well as group by time interaction effects, were evaluated. Statistical significance was set at an alpha of 0.05. Partial eta-square $\left(\eta_{p}^{2}\right)$ was calculated as an estimate of effect size.

\section{Results}

Table 1 displays the demographic characteristics of the analyzed sample. The mean (SD) age of the entire sample was 21.3 (1.6) yrs, with the sample being predominately female (60\%). There were no statistically significant differences for the demographic or behavioral characteristics across the 3 conditions; however, gender approached statistical significance $(P=0.05)$. 
Table 1 Characteristics of the sample.

\begin{tabular}{|l|l|l|l|l|}
\hline Variable & Interference + Exercise & Interference Only & Control & P-Value \\
\hline $\mathrm{N}$ & 17 & 16 & 17 & \\
\hline Age, mean years & $21.1(1.6)$ & $21.8(2.1)$ & $21.1(1.1)$ & 0.36 \\
\hline Gender, \% Female & 41.2 & 56.3 & 82.4 & 0.05 \\
\hline Race, \% non-Hispanic white & 94.1 & 62.5 & 76.5 & 0.23 \\
\hline BMI, mean kg/m ${ }^{2}$ & $24.5(3.1)$ & $26.8(6.4)$ & $24.1(3.3)$ & 0.19 \\
\hline MVPA, mean min/week & $224.1(172.9)$ & $185.6(131.2)$ & $160.3(68.4)$ & 0.37 \\
\hline
\end{tabular}

BMI, body mass index

MVPA, moderate-to-vigorous physical activity

Values in parentheses are standard deviations

An ANOVA was used to calculate the P-values for the continuous variables (e.g., age), whereas a chi-square analysis was used for the categorical variables (e.g., gender).

Table 2 displays the physiological (heart rate) responses to the exercise and control conditions. There was a statistically significant time by group interaction $\left(F=111.3, P<0.001, \eta_{p}^{2}=0.83\right)$. That is, non-exercise conditions (Interference Only and Control) had steady heart rates over the 20-minute period (prior to cognitive assessment), while the exercise condition increased their heart rate from 76.7 (9.2) to 130.4 (14.1) bpm.

Table 2 Heart rate responses across the three conditions.

\begin{tabular}{|c|c|c|c|c|}
\hline Variable & Interference + Exercise & Interference Only & Control & Test-Statistic \\
\hline $\mathrm{N}$ & 17 & 16 & 17 & \\
\hline $\begin{array}{l}\text { Baseline heart rate, } \\
\text { mean bpm }\end{array}$ & 76.7 (9.2) & $73.3(12.7)$ & $70.1(14.5)$ & \multirow{2}{*}{$\begin{array}{l}F(\text { time })=69.9, P<.001, \\
\eta_{p}^{2}=0.60 \\
F(\text { group })=61.4, P<.001, \\
\eta_{p}^{2}=0.73 \\
F(\text { time } x \text { group })=111.3, \\
P<.001, \eta_{p}^{2}=0.83\end{array}$} \\
\hline $\begin{array}{l}\text { Endpoint heart rate, } \\
\text { mean bpm }\end{array}$ & 130.4 (14.1) & $68.2(10.8)$ & $67.4(9.4)$ & \\
\hline
\end{tabular}

Values in parentheses are standard deviations.

Table 3 displays the cognitive scores across the experimental conditions. There was a significant main effect for Stroop type $\left(F=34.5, P<.004, \eta_{p}^{2}=0.45\right)$, a significant main effect for group $(F=3.25$, $\left.P=.04, \eta_{p}^{2}=0.13\right)$, and a significant Stroop by group interaction $\left(F=2.67, P=.03, \eta_{p}^{2}=0.11\right)$. Across the three conditions, there was no difference for Stroop-congruent $\left(F=1.13, P=0.33, \eta_{p}^{2}=0.05\right)$ or Stroopcontrol $\left(F=2.84, P=0.07, \eta_{p}^{2}=0.12\right)$, but Stroop-incongruent was significant $\left(F=4.70, P=0.01, \eta_{p}^{2}=0.18\right)$. 
For Stroop-incongruent, Interference + Exercise was not different than Interference Only $(\mathrm{P}=0.85)$ but was worse than Control $(P=0.002)$. Similarly, Interference Only had a higher (worse) Stroopincongruent score than Control $(P=0.01)$.

Table 3 Cognitive scores across the experimental conditions.

\begin{tabular}{|c|c|c|c|c|}
\hline Variable & Interference + Exercise & Interference Only & Control & Test-Statistic \\
\hline $\mathrm{N}$ & 17 & 16 & 17 & \\
\hline \multicolumn{5}{|l|}{ Stroop, mean ms } \\
\hline Congruent & $890.0(157.0)$ & $928.0(209.0)$ & $837.0(139.1)$ & \multirow{2}{*}{$\begin{array}{l}F(\text { Stroop })=34.5, \\
P<.001, \eta_{p}^{2}=0.45\end{array}$} \\
\hline Incongruent & $1193.0(276.0)$ & 1172.0 (348.9) & $929.0(137.4)$ & \\
\hline Control & 948.9 (140.5) & $955.2(178.1)$ & $830.7(167.1)$ & $\begin{array}{l}F(\text { group })=3.25, \\
P=.04, \eta_{p}^{2}=0.13 \\
F(\text { Stroop } x \\
\text { group) }=2.67, P=.03, \\
\eta_{p}^{2}=0.11\end{array}$ \\
\hline \multicolumn{5}{|c|}{ Memory, mean \# of words } \\
\hline Immediate Recall & $4.70(1.7)$ & $3.93(1.3)$ & $7.76(2.4)$ & $\begin{array}{l}F(\text { time })=53.3, \\
P<0.001, \eta_{p}^{2}=0.53\end{array}$ \\
\hline Delayed Recall & $3.23(1.4)$ & $2.68(1.1)$ & $6.41(3.4)$ & $\begin{array}{l}F(\text { group })=16.7, \\
P<0.001, \eta_{p}^{2}=0.41 \\
F(\text { time } x \\
\text { group) }=0.12, \\
P=0.89, \eta_{p}^{2}=0.01\end{array}$ \\
\hline
\end{tabular}

Values in parentheses are standard deviations.

Mean memory scores are also displayed in Table 3, with individual immediate memory recall and delayed memory recall scores, respectively, shown in Figure 2 and Figure 3. For the memory assessment, there was a significant main effect for time $\left(F=53.3, P<0.001, \eta_{p}^{2}=0.53\right)$, main effect for group $\left(F=16.7, P<0.001, \eta_{p}^{2}=0.41\right)$, but no time by group interaction $\left(F=0.12, P=0.89, \eta_{p}^{2}=0.01\right)$. The Control condition (i.e., no interference and no exercise) had significantly higher immediate memory (7.76 words) when compared to the Interference + Exercise ( $P=0.0003 ; 4.70$ words) and Interference Only ( $P<0.0001 ; 3.93$ words) groups. Although the Interference + Exercise (4.70 words) recalled more words than the Interference Only (3.93 words) group for the immediate recall, this was not statistically significant $(P=0.18)$. Similar results occurred for delayed memory recall. That is, the Control group recalled more words than the Interference + Exercise $(P=0.001)$ and Interference Only 
groups $(P=0.0003)$, but there was no statistically significant difference between Interference + Exercise and Interference Only ( $P=0.24)$.

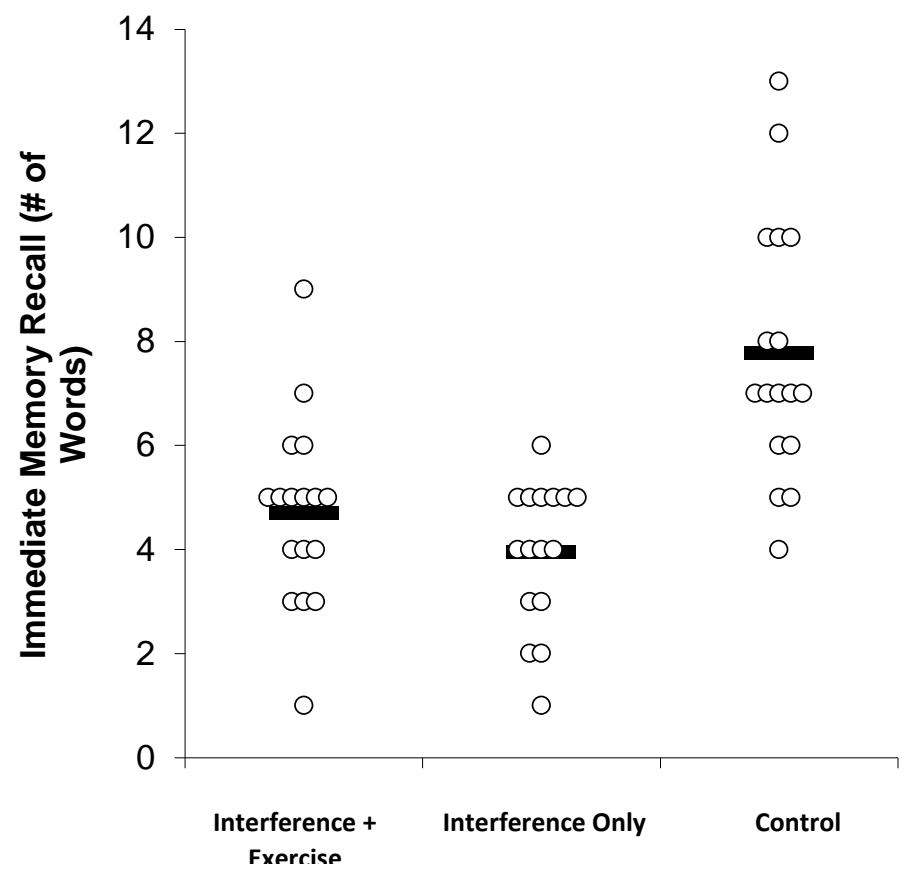

Figure 2 Immediate memory recall across the 3 conditions.

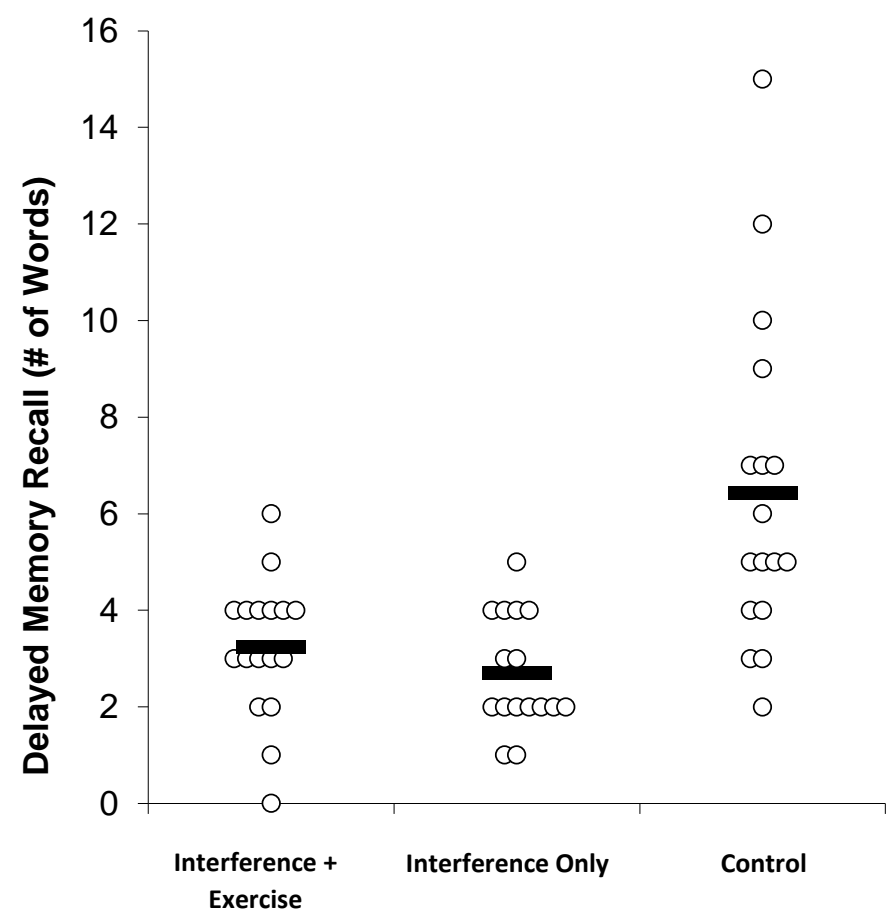

Figure 3 Delayed memory recall across the 3 conditions. 


\section{Discussion}

Only three published experiments, to date, have examined the effects of acute exercise on either proactive and/or retroactive cognitive-related memory interference [12-14]. The present experiment extends this emerging line of inquiry by evaluating whether acute moderate-intensity exercise can attenuate a concurrent memory interference effect. The main findings from this experiment are as follows. We demonstrated evidence of a concurrent memory interference effect, as the control group (i.e., no concurrent interference) performed significantly better on the Stroop-incongruent task and on both the immediate and delayed episodic memory assessments. Regarding whether acute exercise could attenuate a concurrent memory interference effect, our results provided some evidence to suggest this may be possible. That is, the Interference + Exercise (4.70 words) group recalled more words than the Interference Only (3.93 words) for the immediate memory recall. Results were similar for the delayed memory recall assessment (3.23 vs. 2.68 words). However, these differences were not statistically significant.

As indicated in other work, acute moderate-intensity exercise may help to facilitate prefrontal cortex functioning. For example, Tsujii et al. [35] demonstrate that acute moderate-intensity exercise improved prefrontal cortex-dependent memory function and increased cerebral oxygenation in the prefrontal cortex. Other work demonstrates that acute moderate-intensity exercise increases neural activity in the prefrontal cortex [36]. One potential explanation for our null exercise interference findings is that, perhaps, our intensity level was too low [37]. Emerging work demonstrates that highintensity acute exercise improves prefrontal cortex function [38]. Our recent empirical work [1] and review [39] provides support for high-intensity exercise on memory function. Further, animal work demonstrates that a single bout of high-intensity exercise can induce BDNF-TrkB signaling in the prefrontal cortex [40], which we have thoroughly discussed elsewhere as playing an important role in memory function [41]. Another potential explanation for our observed null findings may be from the absence of a sleep period (to facilitate memory consolidation) between memory encoding and recall [42]. Further, there was a greater proportion of females in our control group, which could have contributed to our null exercise interference findings. As we have demonstrated elsewhere [43], females tend to outperform males on verbal memory tasks; however, as we recently demonstrated [44], gender does not appear to moderate the effects of acute exercise on memory function.

Limitations of this study include the relatively small homogenous sample, which may limit our study's generalizability and statistical power. Thus, future work should consider expanding this line of inquiry to other, more diverse populations. Such work should also consider evaluating a bout of higher-intensity exercise, which may have a greater effect on attenuating concurrent memory interference.

In conclusion, this novel experimental study demonstrates that we were effective in inducing concurrent memory interference. We did not, however, demonstrate statistical evidence that acute moderate-intensity exercise attenuates a concurrent memory interference effect. 


\section{Acknowledgments}

We have no conflicts of interest and no funding was used to prepare this manuscript.

\section{Author Contributions}

Author H.L. collected the data. Authors H.L. and L.C. provided feedback on the manuscript. Author P.L. supervised the project and drafted the manuscript.

\section{Competing Interests}

The authors have declared that no competing interests exist.

\section{References}

1. Frith E, Sng E, Loprinzi PD. Randomized controlled trial evaluating the temporal effects of highintensity exercise on learning, short-term and long-term memory, and prospective memory. Eur J Neurosci. 2017; 46: 2557-2564. doi:10.1111/ejn.13719

2. Haynes IV JT, Frith E, Sng E, Loprinzi PD. Experimental effects of acute exercise on episodic memory function: Considerations for the timing of exercise. Psychol Rep. 2018, 1: 33294118786688. doi:10.1177/0033294118786688

3. Siddiqui A, Loprinzi PD. Experimental investigation of the time course effects of acute exercise on false episodic memory. J Clin Med. 2018; 7, pii: E157. doi:10.3390/jcm7070157

4. Sng E, Frith E, Loprinzi PD. Experimental effects of acute exercise on episodic memory acquisition: Decomposition of multi-trial gains and losses. Physiol Behav. 2018; 186: 82-84. doi:10.1016/j.physbeh.2018.01.014

5. Sng E, Frith E, Loprinzi PD. Temporal effects of acute walking exercise on learning and memory function. Am J Health Promot. 2018; 32: 1518-1525. doi:10.1177/0890117117749476

6. Tulving E. Episodic and semantic memory. In: Tulving E, Donaldson W, eds. Organization of memory. Oxford, England: Academic Press; 1972.

7. Loprinzi PD, Edwards MK, Frith E. Potential avenues for exercise to activate episodic memoryrelated pathways: A narrative review. Eur J Neurosci. 2017; 46: 2067-2077. doi:10.1111/ejn.13644

8. Loprinzi PD, Ponce P, Frith E. Hypothesized mechanisms through which acute exercise influences episodic memory. Physiol Int. 2018; 105: 285-297. doi:10.1556/2060.105.2018.4.28

9. Frith E, Loprinzi PD. Physical activity and individual cognitive funcion parameters: Unique exercise-induced mechansims. J Cogn-Behav Psychother Res. 2018; 7: 92-106.

10. Loprinzi PD. The effects of exercise on long-term potentiation: A candidate mechanism of the exercise-memory relationship. OBM Neurobiol. 2019; 3: 13.

11. Guise KG, Shapiro ML. Medial prefrontal cortex reduces memory interference by modifying hippocampal encoding. Neuron. 2017; 94: 183-192. e188. doi:10.1016/j.neuron.2017.03.011 
OBM Integrative and Complementary Medicine 2019; 4(2), doi:10.21926/obm.icm.1902039

12. Frith E, Sng E, Loprinzi PD. Randomized controlled trial considering varied exercises for reducing proactive memory interference. J Clin Med. 2018, 7, pii: E147. doi:10.3390/jcm7060147

13. Haynes IV JT, Loprinzi PD. Acute cardiovascular exercise on proactive memory interference. J Cogn Enhanc. 2019; 3: 139-143. doi:https://doi.org/10.1007/s41465-018-0101-4

14. Wingate S, Crawford L, Frith E, Loprinzi PD. Experimental investigation of the effects of acute exercise on memory interference. Health Promot Perspect. 2018; 8: 208-214. doi:10.15171/hpp.2018.28

15. Jo JS, Chen J, Riechman S, Roig M, Wright DL. The protective effects of acute cardiovascular exercise on the interference of procedural memory. Psychol Res. 2018; 10. doi:10.1007/s00426-018-1005-8

16. Roig M, Ritterband-Rosenbaum A, Lundbye-Jensen J, Nielsen JB. Aging increases the susceptibility to motor memory interference and reduces off-line gains in motor skill learning. Neurobiol Aging. 2014; 35: 1892-1900. doi:10.1016/j.neurobiolaging.2014.02.022

17. Hung CL, Tseng JW, Chao HH, Hung TM, Wang HS. Effect of acute exercise mode on serum brain-derived neurotrophic factor (bdnf) and task switching performance. J Clin Med. 2018; 7: 301. doi:10.3390/jcm7100301

18. Siddiqui S, Chatterjee U, Kumar D, Siddiqui A, Goyal N. Indian J Psychiatry. 2008; 50: 202-208.

19. Erickson KI, Leckie RL, Weinstein AM. Physical activity, fitness, and gray matter volume. Neurobiol Aging. 2014; 35 Suppl 2: S20-28. doi:10.1016/j.neurobiolaging.2014.03.034

20. Tillman B, Loprinzi PD. The experimental effects of acute exercise intensity on episodic memory and working memory function. J Neurobehav Sci. 2019; 6: 12-20.

21. Jubelt LE, Barr RS, Goff DC, Logvinenko T, Weiss AP, Evins AE. Effects of transdermal nicotine on episodic memory in non-smokers with and without schizophrenia. Psychopharmacology (Berl). 2008; 199: 89-98. doi:10.1007/s00213-008-1133-8

22. Klaming R, Annese J, Veltman DJ, Comijs HC. Episodic memory function is affected by lifestyle factors: a 14-year follow-up study in an elderly population. Neuropsychol Dev Cogn B Aging Neuropsychol Cogn. 2016; 24: 528-542. doi:10.1080/13825585.2016.1226746

23. Henry JD, Rendell PG. A review of the impact of pregnancy on memory function. J Clin Exp Neuropsychol. 2007; 29: 793-803. doi:10.1080/13803390701612209

24. Labban JD, Etnier JL. Effects of acute exercise on long-term memory. Res Q Exerc Sport. 2011; 82: 712-721. doi:10.1080/02701367.2011.10599808

25. Sherman SM, Buckley TP, Baena E, Ryan L. Caffeine enhances memory performance in young adults during their non-optimal time of day. Front Psychol. 2016; 7: 1764. doi:10.3389/fpsyg.2016.01764

26. Wammes JD, Good TJ, Fernandes MA. Autobiographical and episodic memory deficits in mild traumatic brain injury. Brain Cogn. 2017; 111: 112-126. doi:10.1016/j.bandc.2016.11.004

27. Hindocha C, Freeman TP, Xia JX, Shaban NDC, Curran HV. Acute memory and psychotomimetic effects of cannabis and tobacco both 'joint' and individually: A placebo-controlled trial. Psychol Med. 2017, 47: 2708-2719. doi:10.1017/S0033291717001222 
28. Le Berre AP, Fama R, Sullivan EV. Executive functions, memory, and social cognitive deficits and recovery in chronic alcoholism: A critical review to inform future research. Alcohol Clin Exp Res. 2017; 41: 1432-1443. doi:10.1111/acer.13431

29. Garber CE, Blissmer B, Deschenes MR, et al. American College of Sports Medicine position stand. Quantity and quality of exercise for developing and maintaining cardiorespiratory, musculoskeletal, and neuromotor fitness in apparently healthy adults: Guidance for prescribing exercise. Med Sci Sports Exerc. 2011; 43: 1334-1359. doi:10.1249/MSS.0b013e318213fefb

30. McNerney MW, Radvansky GA. Mind racing: The influence of exercise on long-term memory consolidation. Memory. 2015; 23: 1140-1151. doi:10.1080/09658211.2014.962545

31. Geffen GM, Butterworth P, Geffen LB. Test-retest reliability of a new form of the auditory verbal learning test (AVLT). Arch Clin Neuropsychol. 1994; 9: 303-316.

32. Audenaert $\mathrm{K}$, Lahorte $\mathrm{P}, \mathrm{Brans} \mathrm{B}$, et al. The classical stroop interference task as a prefrontal activation probe: A validation study using 99Tcm-ECD brain SPECT. Nucl Med Commun. 2001; 22: 135-143.

33. Vora JP, Varghese R, Weisenbach SL, Bhatt T. Test-retest reliability and validity of a customdesigned computerized neuropsychological cognitive test battery in young healthy adults. J Psychol Cogn. 2016; 1: 11-19.

34. Ball TJ, Joy EA, Gren LH, Shaw JM. Concurrent validity of a self-reported physical activity "vital sign" questionnaire with adult primary care patients. Prev Chronic Dis. 2016; 13: E16. doi:10.5888/pcd13.150228

35. Tsujii T, Komatsu K, Sakatani K. Acute effects of physical exercise on prefrontal cortex activity in older adults: a functional near-infrared spectroscopy study. Adv Exp Med Biol. 2013; 765: 293-298. doi:10.1007/978-1-4614-4989-8_41

36. Li L, Men WW, Chang YK, Fan MX, Ji L, Wei GX. Acute aerobic exercise increases cortical activity during working memory: a functional MRI study in female college students. PloS one. 2014, 9, e99222. doi:10.1371/journal.pone.0099222

37. Thomas R, Johnsen LK, Geertsen SS, et al. Acute exercise and motor memory consolidation: the role of exercise intensity. PloS one. 2016; 11: e0159589. doi:10.1371/journal.pone.0159589

38. Basso JC, Shang A, Elman M, Karmouta R, Suzuki WA. Acute exercise improves prefrontal cortex but not hippocampal function in healthy adults. J Int Neuropsychol Soc. 2015 ; 21: 791801. doi:10.1017/S135561771500106X

39. Loprinzi PD. Intensity-specific effects of acute exercise on human memory function: considerations for the timing of exercise and the type of memory. Health Promot Perspect. 2018; 8: 255-262. doi:10.15171/hpp.2018.36

40. Baranowski BJ, MacPherson REK. Acute exercise induced BDNF-TrkB signalling is intact in the prefrontal cortex of obese, glucose-intolerant male mice. Appl Physiol Nutr Metab. 2018; 43: 1083-1089. doi:10.1139/apnm-2018-0108

41. Loprinzi PD, Frith E. A brief primer on the mediational role of BDNF in the exercise-memory link. Clin Physiol Funct Imaging. 2018; 39: 9-14. doi:10.1111/cpf.12522 
42. Dal Maso F, Desormeau B, Boudrias MH, Roig M. Acute cardiovascular exercise promotes functional changes in cortico-motor networks during the early stages of motor memory consolidation. Neurolmage. 2018; 174: 380-392. doi:10.1016/j.neuroimage.2018.03.029

43. Loprinzi PD, Frith E. The role of sex in memory function: Considerations and recommendations in the context of exercise. J Clin Med. 2018, 7, pii: E132. doi:10.3390/jcm7060132

44. Johnson L, Loprinzi PD. The effects of acute exercise on episodic memory function among young University students: Moderation considerations by biological sex. Brain Sci. 2019; 9, pii: E87.

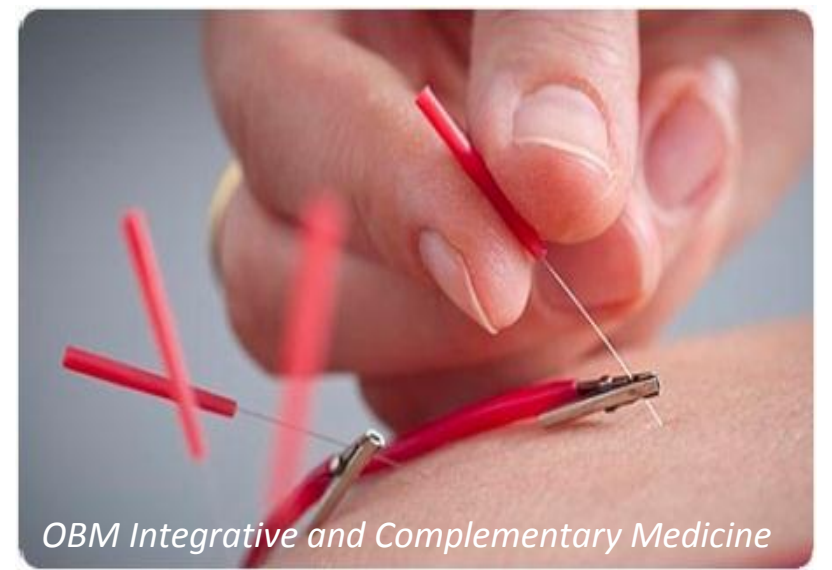

Enjoy OBM Integrative and Complementary Medicine by:

1. Submitting a manuscript

2. Joining in volunteer reviewer bank

3. Joining Editorial Board

4. Guest editing a special issue

For more details, please visit:

http://www.lidsen.com/journals/icm 